\title{
Dipolar ordering in a molecular nanomagnet detected using muon spin relaxation
}

\author{
F. L. Pratt, ${ }^{1}$ E. Micotti, ${ }^{2}$ P. Carretta, ${ }^{3}$ A. Lascialfari, ${ }^{3,4}$ P. Arosio, ${ }^{4}$ T. Lancaster, ${ }^{5}$ S. J. Blundell, ${ }^{6}$ and A. K. Powell ${ }^{7,8}$ \\ ${ }^{1}$ ISIS Facility, STFC Rutherford Appleton Laboratory, Chilton, Oxfordshire OX11 OQX, United Kingdom \\ ${ }^{2}$ Istituto di Ricerche Farmacologiche "Mario Negri" - IRCCS" - I-20156 Milano, Italy \\ ${ }^{3}$ Dipartimento di Fisica, Università di Pavia and CNISM, I-127100 Pavia, Italy \\ ${ }^{4}$ Dipartimento di Fisica and INSTM, Università degli studi di Milano, I-20133 Milano, Italy \\ ${ }^{5}$ Centre for Materials Physics, Department of Physics, Durham University, Durham DH1 3LE, United Kingdom \\ ${ }^{6}$ Department of Physics, University of Oxford, Clarendon Laboratory, Parks Road, Oxford OX1 3PU, United Kingdom \\ ${ }^{7}$ Institute of Inorganic Chemistry, Karlsruhe Institute of Technology, Engesserstrasse 15, D76131 Karlsruhe, Germany \\ ${ }^{8}$ Institute of Nanotechnology, Karlsruhe Institute of Technology, Hermann-von-Helmholtz-Platz 176344, \\ Eggenstein-Leopoldshafen, Germany \\ (Received 31 October 2013; revised manuscript received 14 March 2014; published 21 April 2014)
}

\begin{abstract}
Implanted muons have been used as a local probe to detect the magnetic ordering in the molecular magnetic nanodisk system $\mathrm{Fe}_{19}$. Two distinct groups of muon sites are identified from the relaxation data, reflecting sites near the magnetic core and sites distributed over the rest of the molecule. Dipole field calculations and Monte Carlo simulations confirm that the observed transition in $\mathrm{Fe}_{19}$ is consistent with magnetic ordering driven by interactions between molecules that are predominantly dipolar in nature. The triclinic crystal structure of this system gives the dipolar field a significant component transverse to the easy spin axis and the parallel component provides a dipolar bias closely tuned to the first level crossing of the system. These factors enhance the quantum tunneling between levels, thus enabling the system to avoid spin freezing at low temperatures and efficiently reach the dipolar ordered state.
\end{abstract}

DOI: 10.1103/PhysRevB.89.144420

\section{INTRODUCTION}

Single molecule magnets (SMMs) [1,2] continue to attract interest both from the viewpoint of fundamental science and in terms of their potential applications in areas such as data storage and quantum computing. An important limiting factor for such applications is the effect of finite intermolecular interactions that can lead to decoherence of the spin state [3] and hence loss of information. In particular, weak residual dipolar interactions between the spins on different molecules are always present for molecules with finite spin and such interactions can ultimately lead to dipolar ordering at low temperatures [4,5].

For many highly anisotropic SMM systems, however, spin freezing takes place at low temperatures and the equilibrium dipolar ordered ground state is not reached without careful annealing in a magnetic field chosen to enhance the magnetic quantum tunneling rates [5]. The $\mathrm{Fe}_{19} \mathrm{SMM}$ system [6] is an interesting example of an SMM that has a combination of high spin and moderate anisotropy, thus making it a good candidate to observe dipolar-driven long-range magnetic ordering. The magnetic core of the molecule has a disklike geometry (Fig. 1), so it can be described as a magnetic nanodisk. Two variants have been reported [6], having the same core but slightly different ligands: the metheidi form, labeled here as $\mathrm{Fe}_{19}(\mathrm{~m})$, has the more tightly packed crystal structure, whereas the etheidi form, labeled here as $\mathrm{Fe}_{19}(\mathrm{e})$,

Published by the American Physical Society under the terms of the Creative Commons Attribution 3.0 License. Further distribution of this work must maintain attribution to the author(s) and the published article's title, journal citation, and DOI.
PACS number(s): 75.50.Xx, 76.75.+i, 75.25.-j, 75.40.Cx

has a structure with approximately $10 \%$ more volume per molecule. Both structures are triclinic and it is found that this low symmetry has an important bearing on the dipolar-driven magnetic properties.

Although an analysis of the experimental magnetic susceptibility data originally suggested a spin value of $33 / 2$ for the $\mathrm{Fe}_{19}$ family [6], a subsequent computational study of exchange couplings within the Fe cluster [7] suggested that the Fe spins take the ferrimagnetic arrangement shown in Fig. 1 with a total spin value of $S=35 / 2$. This was confirmed by a recent single crystal EPR study [8] where the easy axis was determined to lie within the main plane of the disk as shown in Fig. 1. The variation of the muon spin relaxation $(\mu \mathrm{SR})$ with temperature $T$ in $\mathrm{Fe}_{19}$ (e) was previously measured by some of us down to $2 \mathrm{~K}[9,10]$. No indication of magnetic ordering was found in this $T$ range, but the relaxation rate was found to flatten off at low temperatures, in common with the behavior seen in other SMMs $[2,11,12]$. Long-range magnetic ordering of $\mathrm{Fe}_{19}$ was first revealed by specific heat $[13,14]$, with ordering peaks occurring at $1.07 \mathrm{~K}$ for $\mathrm{Fe}_{19}(\mathrm{e})$ and at $1.19 \mathrm{~K}$ for $\mathrm{Fe}_{19}(\mathrm{~m})$. Further evidence for an ordered state was provided by NMR relaxation [15], which showed a $1 / T_{1}$ relaxation below $1 \mathrm{~K}$ that could be assigned to magnons. In the study reported here we have extended the $\mu \mathrm{SR}$ measurements to much lower $T$ and have observed changes in the muon spin relaxation taking place around $1.2 \mathrm{~K}$ for $\mathrm{Fe}_{19}(\mathrm{~m})$ that are consistent with the ordering inferred from the previous measurements.

In the original report of magnetic ordering measured by specific heat in $\mathrm{Fe}_{19}(\mathrm{~m})$ and $\mathrm{Fe}_{19}(\mathrm{e})$ by Affronte et al. [13], it was questioned whether the ordering in $\mathrm{Fe}_{19}$ is predominantly dipolar-driven, since their estimate of the dipolar interaction energy [16] was only $190 \mathrm{mK}$ and they suggested that superexchange interactions mediated via the water of crystallisation surrounding each cluster must be driving the ordering. 


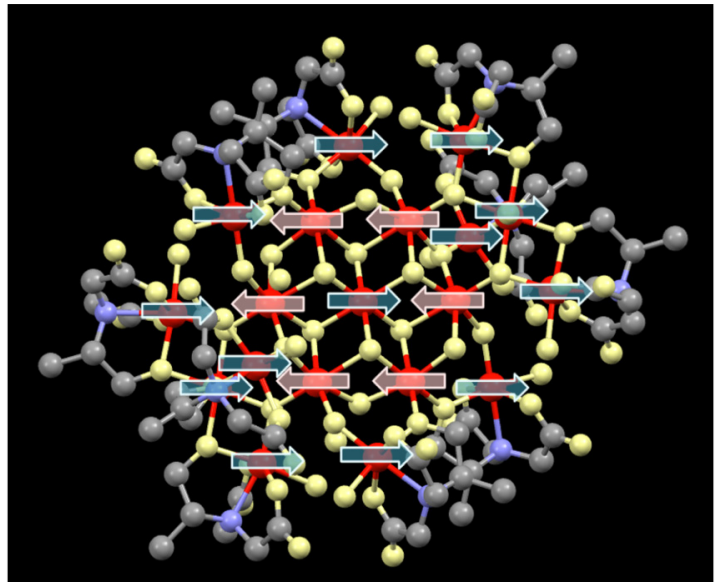

FIG. 1. (Color online) The ferrimagnetic spin arrangement of $S=5 / 2 \mathrm{Fe}$ atoms within the $\mathrm{Fe}_{19}$ molecule leading to the $S=35 / 2$ ground state (the easy axis is within the plane of the disk pointing in the direction shown by the central spin [8]).

However, from more detailed consideration of the exact crystal structure and known easy axis orientation, we find that the observed ordering temperature in $\mathrm{Fe}_{19}(\mathrm{~m})$ is fully consistent with intermolecular interactions that are predominantly dipolar in nature. We also find that the dipolar field "bias" produced at one molecule by its neighbors has an important influence on the ability of the molecule to reach the equilibrium dipolar ordered state and the specific case of $\mathrm{Fe}_{19}$ is particularly favorable in this respect.

\section{EXPERIMENTAL DATA}

A polycrystalline sample of $\mathrm{Fe}_{19}(\mathrm{~m})$, full formula $\mathrm{Fe}_{19} \mathrm{O}_{109} \mathrm{~N}_{11} \mathrm{C}_{70} \mathrm{H}_{186}$, was prepared for the $\mu \mathrm{SR}$ measurements, which were carried out on the ARGUS instrument at the ISIS Neutron and Muon Source. The sample was cooled using a ${ }^{3} \mathrm{He}$ sorption cryostat giving a base temperature of $0.29 \mathrm{~K}$ and zero field muon spin relaxation measurements were made between this temperature and $1.8 \mathrm{~K}$. A comparison of the relaxation data above and below the transition is shown in Fig. 2. As in previous studies of the temperature dependent $\mu \mathrm{SR}[9,10]$, we observe that a single exponential term does not adequately account for the relaxation. This points towards a broad distribution of couplings of the muon to the magnetic species, due to the multiplicity of possible muon stopping sites in such a large and complex unit cell. A root exponential relaxation function describes the longer time data well, but an additional fast exponential is needed to fully describe the early time relaxation, so the total relaxing asymmetry $a(t)$ is fitted well using the function

$$
a(t)=a_{\mathrm{f}} e^{-\lambda_{\mathrm{f}} t}+a_{\mathrm{s}} e^{-\left(\lambda_{\mathrm{s}} t\right)^{1 / 2}}+a_{\mathrm{bg}},
$$

where $a_{\mathrm{f}}$ and $a_{\mathrm{s}}$ are the amplitudes of the two relaxation components and $\lambda_{\mathrm{f}}$ and $\lambda_{\mathrm{s}}$ their corresponding relaxation rates and $a_{\mathrm{bg}}$ is a background component reflecting muons stopping in the silver sample plate.

In magnetic systems with relatively simple unit cells, the number of muon sites is limited and distinct oscillations are typically observed in the muon relaxation function below the

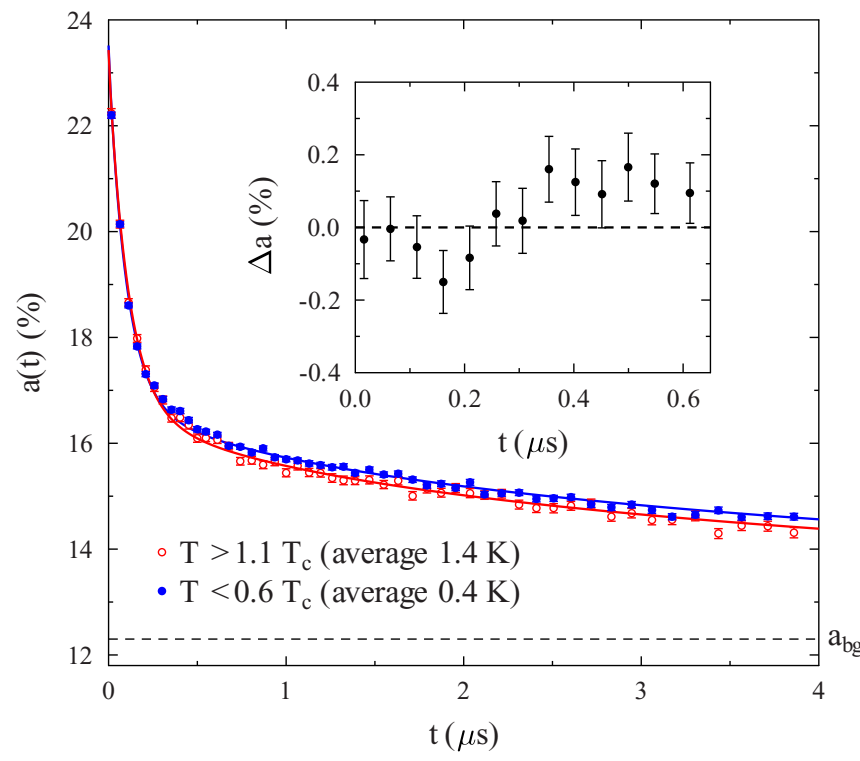

FIG. 2. (Color online) Comparison of zero field $\mu$ SR above and below the $1.2 \mathrm{~K}$ transition. The inset plots the difference between the signals in the two temperature regions, showing that on cooling below $T_{\mathrm{c}}$ the fast component becomes faster and the slow component becomes slower.

magnetic ordering temperature, with many such examples being found in the field of molecular magnets [2]. The frequency of one of these oscillations $f(T)$ tracks the magnetic order parameter $\phi(\mathrm{T})$ with $\beta$ being the relevant critical exponent [17], i.e.,

$$
f(T) / f(0)=\phi(T)=\left(1-T / T_{\mathrm{c}}\right)^{\beta} .
$$

Typically, up to six components can be resolved under favorable conditions [18], but as the complexity of the cell increases, it is expected to become impossible to resolve individual precession components and precession will be replaced by a broad relaxation contribution, whose shape depends on the distribution of fields at the muon stopping sites. The associated relaxation rate is still however expected to reflect the magnetic order parameter. With 395 atoms in the unit cell, the present system is expected to be in this highly broadened regime and it can be confirmed that no oscillations are seen in the data of Fig. 2.

A further difference from simpler magnetic systems is the complex nature of the present $\mathrm{Fe}_{19}$ magnetic unit, which leads to a significant background of fluctuating fields related to the SMM level structure. The change in the local field environment due the intermolecular ordering then becomes a subtle change on top of this background, rather than the dominant appearance of a new static field that is seen in the simpler magnets. The subtle change in the relaxation function on entering the dipolar ordered state is seen in Fig. 2. Closer analysis of the change in relaxation shows that the two relaxation components behave differently on going through the transition. This is demonstrated by the difference plot shown in the inset to Fig. 2, which shows that the fast relaxation component that dominates below $0.3 \mu \mathrm{s}$ has a rate that increases below $T_{\mathrm{c}}$, whereas the slow relaxation component has a rate that decreases below $T_{\mathrm{c}}$. 


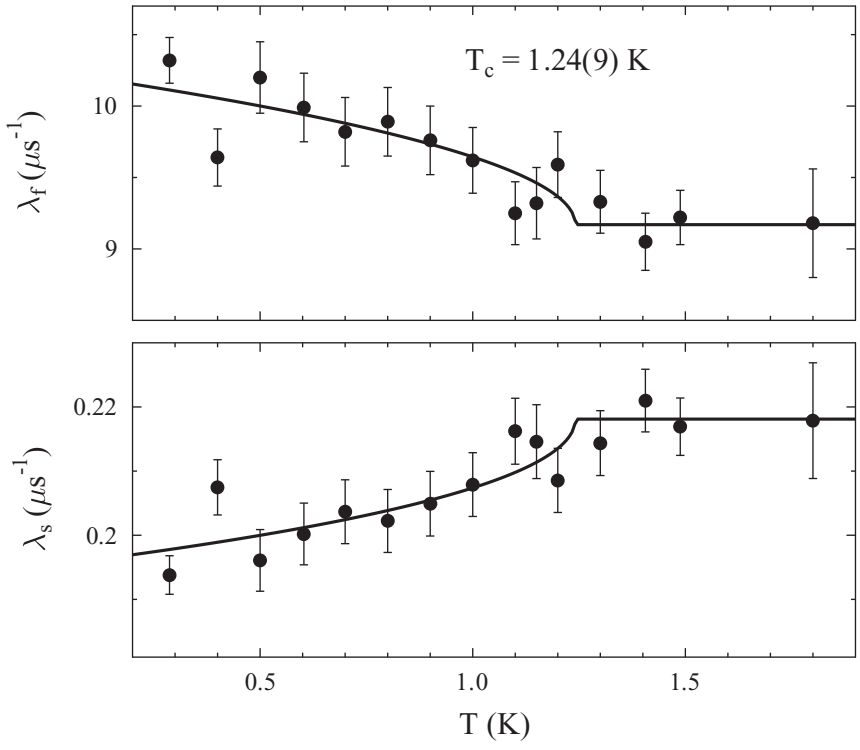

FIG. 3. Temperature dependence of the fast and slow relaxation rates. The changes below $T_{\mathrm{c}}$ can be represented by mean-field $T$ dependence of Eq. (3) (solid lines).

As expected, the changes are not very large, both being of order $10 \%$ when fitted independently.

To minimize the number of independent parameters and express the relaxation rate changes going through the transition in terms of a single $T$ dependent order parameter, we make use of the empirical feature that the product $\lambda_{\mathrm{f}} \lambda_{\mathrm{s}}$ is found to be constant against $T$ by applying this constant product as a constraint in the fitting of both components together. The component amplitudes and the product of the relaxation rates were obtained from a fit to the combined data set averaged over all measured temperatures and these parameters were kept fixed for the detailed analysis of the $T$ dependent relaxation rate. The $T$ dependence of the two (dependent) relaxation rates obtained in this way is shown in Fig. 3. The change in the fast relaxation rate below the transition temperature $\Delta \lambda_{f}$ is well described by an order parameter exponent taking the mean-field value $\beta=0.5$, i.e.,

$$
\Delta \lambda_{\mathrm{f}}(T) / \Delta \lambda_{\mathrm{f}}(0)=\phi(T)=\left(1-T / T_{\mathrm{c}}\right)^{0.5}
$$

and the fit is shown as the solid lines in Fig. 3. The value $T_{\mathrm{c}}=1.24(9) \mathrm{K}$ obtained from this fit is fully consistent with the transition temperature of $1.19 \mathrm{~K}$ previously obtained from the specific heat [13].

\section{DIPOLAR ORDERING MODE AND TRANSITION TEMPERATURE}

\section{A. Dipolar energies}

We now consider whether the observed $T_{\mathrm{c}}$ is reasonable for an ordering transition in this system driven predominantly by dipolar interactions. To investigate further, we have calculated the dipolar field vector $\boldsymbol{B}_{\text {dip }}$ at the center of the molecule due to the surrounding molecules for a range of possible modes of ordering. For these calculations, we replace the alternating spin distribution at the core of the molecule by spin $S=35 / 2$ placed exactly at the center of the molecule, which is a good approximation when considering only intermolecular interactions. For the triclinic structure of this system [6], $\boldsymbol{B}_{\text {dip }}$ is not parallel to the molecular moment $\boldsymbol{m}$, so we calculate the dipolar stabilization energy as $E_{0}=\boldsymbol{m} \cdot \boldsymbol{B}_{\text {dip }}=m B_{\text {dip }} \cos \phi$. When $E_{0}$ is positive, the dipolar ordering mode is stable.

For a simple primitive lattice system with dominant dipolar interactions and spins constrained to lie along an easy axis, there are just eight possible magnetic ordering modes [16]. In Table I, the dipolar energies of these different magnetic structures are listed for the two $\mathrm{Fe}_{19}$ molecules, along with $\mathrm{Fe}_{8}$ [19] for comparison.

For both $\mathrm{Fe}_{19}$ systems, the $(0,0,1 / 2)$ ordering with ferromagnetic (FM) arrangement along $a$ and $b$ and antiferromagnetic (AF) arrangement along $c$ is strongly favored compared to the other possibilities for overall AF order. The ordering pattern of the most stable structure for $\mathrm{Fe}_{19}(\mathrm{~m})$ is illustrated in Fig. 4.

It is interesting to compare $\mathrm{Fe}_{19}$ with the well studied $\mathrm{Fe}_{8}$ system. For $\mathrm{Fe}_{8}$, the most stable ordering is again the $(0,0$, $1 / 2)$ mode (Table I), but it is notable that the $(0,0,1 / 2)$, $(0$, $1 / 2,0)$, and $(0,1 / 2,1 / 2)$ ordering modes have almost the same energy, which indicates that the $b c$ plane dipolar interactions are highly frustrated in this system. It would therefore perhaps be better to regard the $\mathrm{Fe}_{8}$ system as a set of magnetic chains that are relatively strongly FM coupled along the $a$ axis but with weak effective coupling in the transverse directions. This situation leads to a significant suppression of $T_{\mathrm{c}}$ with respect to the underlying coupling, as is generally seen in magnetic chain systems $[2,20]$.

A further comparison can be made with the trigonal $\mathrm{Fe}_{17}$ SMM; this has a more complex structure than the triclinic systems, with six molecules in the unit cell. Dipolar ordering in this phase has been identified from a peak in the specific heat at $0.81 \mathrm{~K}$ [21]. The ordering was verified by neutron scattering to be a commensurate FM state [22], but this study also suggested a higher $T_{\mathrm{c}}$ of $1.13 \mathrm{~K}$ on the basis of the $T$ dependent scattering intensity. The observed FM order in this case is broadly consistent with the dipolar energy (Table I). For monodomain FM particles, $T_{\mathrm{c}}$ is dependent on the demagnetizing factor $N$. The entries for the FM order in Table I are split into a spherical value $(N=1 / 3)$ and a term for the departure from spherical. The two reported $T_{\mathrm{c}}$ values of $0.81 \mathrm{~K}$ [21] and $1.13 \mathrm{~K}$ [22] for $\mathrm{Fe}_{17}$ may be interpreted as corresponding to FM samples with different effective $N$ values.

\section{B. $T_{\mathrm{c}}$ from Monte Carlo simulations}

Although simple mean-field (MF) theory predicts that $T_{\mathrm{c}} / E_{0}=1$, it is expected that the actual ordering temperature ratio for a given structure may vary considerably from this. We have therefore carried out Monte Carlo (MC) simulations on a $20 \times 20 \times 20$ array of spins with periodic boundary conditions to determine the typical degree of departure of the $T_{\mathrm{c}} / E_{0}$ ratio from the MF value for lattices similar to that of $\mathrm{Fe}_{19}$. This approach also allows us to estimate more precise ordering temperatures for our specific structures, which allows us also to assess quantitatively the size of any possible contribution from superexchange that operates alongside the dipolar interaction. In the calculations we include dipolar terms from spins within a sphere large enough to give a total dipolar energy that is within 
TABLE I. The upper part of the table lists dipolar stabilisation energies $E_{0}$ in units of degrees Kelvin for the possible ordering vectors, comparing the two $\mathrm{Fe}_{19}$ systems with $\mathrm{Fe}_{8}$ and the trigonal phase of $\mathrm{Fe}_{17} . N$ is the demagnetizing factor for the $\mathrm{FM}_{\text {case. }} \mathrm{For}_{\mathrm{Fe}} \mathrm{F}_{19}$ and $\mathrm{Fe}_{8}$ the figures in parentheses show $T_{\mathrm{c}}$ estimated from Monte Carlo simulation of the stable modes of ordering. The most stable ordered structure in each case is highlighted in bold. For $\mathrm{Fe}_{17}$, the ordering type was previously reported to be collinear FM from neutron scattering [22]. The lower section lists the measured $T_{\mathrm{c}}$, Weiss temperature, ground-state molecular spin, magnetic anisotropy, easy axis, molecular volume, zero field separation of the lowest two levels in the $S_{z}$ manifold $E_{\text {sep }}=D(2 S-1)$ and $\phi$, the angle between the moment and the dipolar field in the most stable state.

\begin{tabular}{|c|c|c|c|c|}
\hline Space group ( $Z$ molecules per cell) & $\begin{array}{c}\mathrm{Fe}_{19}(\mathrm{~m}) \\
P \overline{1}(Z=1)[6]\end{array}$ & $\begin{array}{c}\mathrm{Fe}_{19}(\mathrm{e}) \\
P \overline{1}(Z=1)[6]\end{array}$ & $\begin{array}{c}\mathrm{Fe}_{8} \\
P 1(Z=1)[19]\end{array}$ & $\begin{array}{c}\mathrm{Fe}_{17} \text { (trigonal) } \\
R \overline{3}(Z=6)[21]\end{array}$ \\
\hline$(0,0,1 / 2) \mathrm{AF}$ & $0.78(0.84)$ & $0.58(0.52)$ & $0.98(0.57)$ & \\
\hline$(0,1 / 2,0) \mathrm{AF}$ & -0.56 & -0.21 & $0.96(0.56)$ & \\
\hline$(0,1 / 2,1 / 2) \mathrm{AF}$ & -0.28 & -0.53 & $0.95(0.48)$ & \\
\hline$(1 / 2,0,0) \mathrm{AF}$ & -0.19 & -0.01 & -0.73 & \\
\hline$(1 / 2,0,1 / 2) \mathrm{AF}$ & 0.13 & 0.00 & -0.75 & \\
\hline$(1 / 2,1 / 2,0) \mathrm{AF}$ & -0.09 & $0.16(0.05)$ & -0.74 & \\
\hline$(1 / 2,1 / 2,1 / 2) \mathrm{AF}$ & $0.21(0.07)$ & 0.00 & -0.68 & \\
\hline$(0,0,0) \mathrm{FM}$ (for sphere with $N=1 / 3$ ) & $0.59(0.20)$ & $0.58(0.21)$ & $0.82(0.39)$ & 0.89 \\
\hline FM shape term & $+0.90(1 / 3-N)$ & $+0.82(1 / 3-N)$ & $+0.52(1 / 3-N)$ & $+1.16(1 / 3-N)$ \\
\hline$T_{\mathrm{c}}(\mathrm{K})$ & $1.19[13]$ & $1.07[13]$ & $0.6[5]$ & $0.81[21], 1.13$ [22] \\
\hline$\theta(\mathrm{K})$ & $-1.7[6]$ & $-1.4[6]$ & $-0.5^{\mathrm{a}}$ & $0.9[21]$ \\
\hline$S$ & $35 / 2[8]$ & $35 / 2^{\mathrm{b}}$ & $10[26]$ & $35 / 2[21]$ \\
\hline$D / k_{\mathrm{B}}(\mathrm{K})$ & $0.043[8]$ & $0.043^{\mathrm{b}}$ & $0.29[26]$ & $0.023[21]$ \\
\hline Easy axis & $(1,-0.89,-0.05)[8]$ & $(1,0.8,-0.33)^{b}$ & $(1,-0.05,0)[27]$ & $(0,0,1)[22]$ \\
\hline$V_{M}\left(\AA^{3}\right)$ & $3536[6]$ & $3929[6]$ & $1956[19]$ & $2743[21]$ \\
\hline$E_{\text {sep }} / k_{\mathrm{B}}(\mathrm{K})$ & 1.5 & 1.5 & 5.6 & 0.78 \\
\hline$\phi$ (degrees) & 35 & 60 & 2 & 0 \\
\hline
\end{tabular}

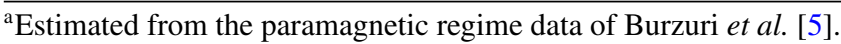

${ }^{\mathrm{b}}$ Assuming the same spin, anisotropy, and easy-axis orientation within the magnetic core as for $\mathrm{Fe}_{19}(\mathrm{~m})$.

$1 \%$ of its asymptotic value, typically around 400 molecules. In Fig. 5, we show the results for a simple rhombohedral lattice in which lattice constants and cell angles are all equal, with the cell angle spanning across a range that encompasses the values for the high-symmetry FCC, SC and BCC lattices. It is clearly

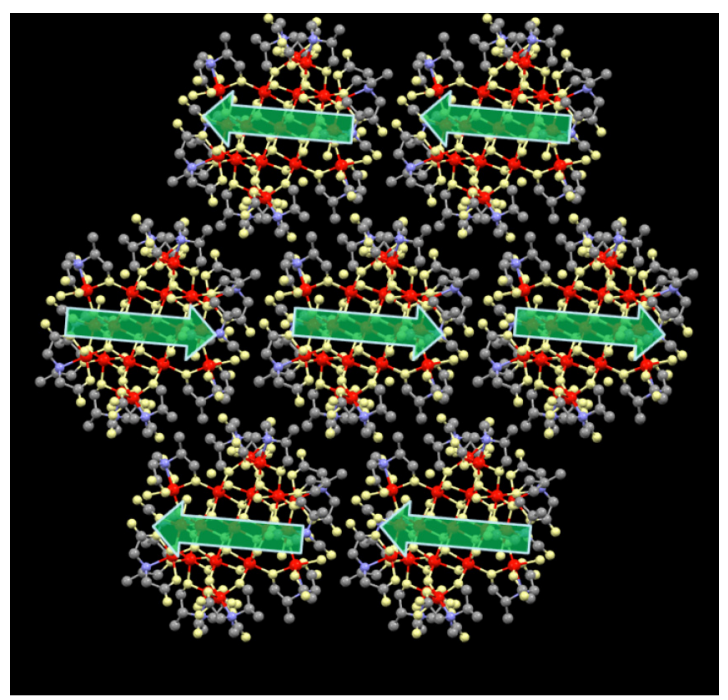

FIG. 4. (Color online) The ordering pattern driven by dipolar interaction for $\mathrm{Fe}_{19}(\mathrm{~m})$, looking down the $a$ axis with the $b$ axis running left to right and the $c$ axis running from the top left molecule to the bottom right molecule. seen from Fig. 5 that the $T_{\mathrm{c}} / E_{0}$ ratio depends sensitively on the orientation of the easy axis, as well as the cell angle.

Calculations of $T_{\mathrm{c}}$ for the specific lattice structures of $\mathrm{Fe}_{19}$ and $\mathrm{Fe}_{8}$ have been made for the set of ordered spin structures that are energetically stable (i.e., positive $E_{0}$ in Table I) and these are listed in parentheses in the upper part of Table I. In each case the $(0,0,1 / 2)$ AF ordering mode that is the most stable on the basis of $E_{0}$ is reconfirmed to be the most stable on the basis of the simulation. Close comparison between the simulated and experimental $T_{\mathrm{c}}$ values and the ground-state energy is made in Table II. For $\mathrm{Fe}_{8}$, the experimental $T_{\mathrm{c}}$ is very well reproduced by the calculation, suggesting that superexchange is insignificant in that structure. However, in the case of $\mathrm{Fe}_{19}$, the calculated $T_{\mathrm{c}}$ is found to be lower than experiment by around $30 \%$ for $\mathrm{Fe}_{19}(\mathrm{~m})$ and $50 \%$ for $\mathrm{Fe}_{19}(\mathrm{e})$. The difference can be represented by including an additional ferromagnetic superexchange contribution $E_{a}=J_{a} S^{2}$ acting along the shortest crystal axis $a$. For $\mathrm{Fe}_{19}(\mathrm{~m})$ where the easy axis orientation has been determined experimentally, taking $E_{a}=0.12 \mathrm{~K}$, exactly reproduces the experimental $T_{\mathrm{c}}$ in the MC simulation. A larger $E_{a}$ value is suggested for $\mathrm{Fe}_{19}(\mathrm{e})$, however, we note that the calculations may not be so reliable here because the easy axis direction has not been specifically determined for this $\mathrm{Fe}_{19}$ variant. In this case, in the absence of any further information, the easy axis was simply assumed to take the same relative orientation within the core as for $\mathrm{Fe}_{19}(\mathrm{~m})$. However, it has been noted that the ligand is expected to strongly influence the anisotropy axis [8], so this is likely to be a poor approximation. Even for $\mathrm{Fe}_{19}(\mathrm{~m})$ the uncertainty of 


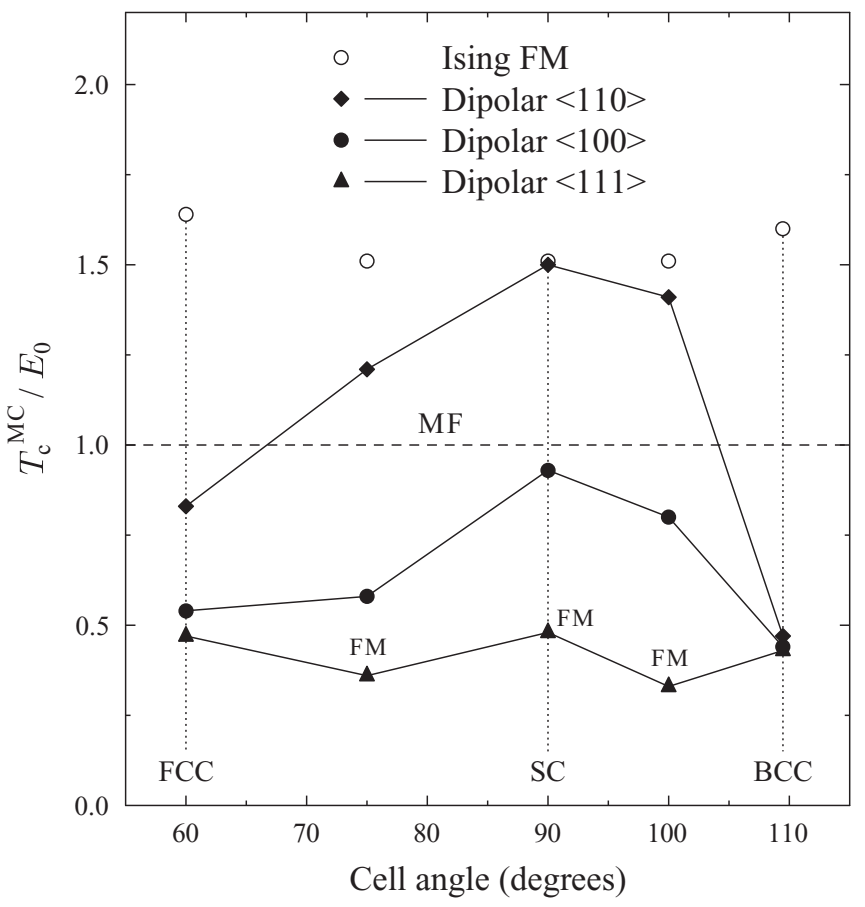

FIG. 5. MC calculation of the ratio of the ordering temperature to the ground-state energy for a simple rhombohedral lattice with different orientations of the easy spin axis. For dipolar interactions, the ordering ratio is highly dependent on the easy axis direction and generally significantly lower than for Ising interactions. The dipolar ordering mode is AF, except for the marked cases with $\langle 111\rangle$ spin orientation.

the easy axis orientation was suggested [8] to be of order $8^{\circ}$ and we note that rotating the easy axis by just $10^{\circ}$ towards the $a$ axis would shift the calculated dipolar transition up to match the experimentally observed value. The values obtained for $E_{a}$ and $J_{a}$ should therefore be regarded as upper limits for the superexchange contribution to the intermolecular coupling. As discussed earlier, the $T_{\mathrm{D}}^{\mathrm{MC}} / E_{0}$ ratio is significantly suppressed for $\mathrm{Fe}_{8}$ due to the effective chainlike character of its structure. In contrast, for the $\mathrm{Fe}_{19}$ systems, the $T_{\mathrm{D}}^{\mathrm{MC}} / E_{0}$ ratio is within $10 \%$ of the MF value (Table II), indicating that the basic MF approximation works reasonably well for these structures.

TABLE II. MC results comparing the $\mathrm{Fe}_{8}$ and $\mathrm{Fe}_{19}$ systems. $T_{\mathrm{c}}$ is the experimentally observed transition, $T_{\mathrm{D}}^{\mathrm{MC}}$ the transition obtained from the MC simulation, and $E_{0}$ the dipolar ground-state energy. In the case of $\mathrm{Fe}_{19}$, a small additional $a$-axis exchange interaction $J_{a}$ provides an additional energy term $E_{a}$ that can reproduce the measured $T_{\mathrm{c}}$.

\begin{tabular}{llcc}
\hline \hline & $\mathrm{Fe}_{8}$ & $\mathrm{Fe}_{19}(\mathrm{~m})$ & $\mathrm{Fe}_{19}(\mathrm{e})$ \\
\hline$T_{\mathrm{c}}(\mathrm{K})$ & 0.6 & 1.19 & 1.07 \\
$T_{\mathrm{D}}^{\mathrm{MC}}(\mathrm{K})$ & 0.57 & 0.84 & 0.52 \\
$E_{0}(\mathrm{~K})$ & 0.98 & 0.78 & 0.58 \\
$T_{\mathrm{D}}^{\mathrm{MC}} / E_{0}$ & 0.58 & 1.08 & 0.90 \\
$E_{a}=J_{a} S^{2}(\mathrm{~K})$ & & 0.12 & 0.17 \\
$J_{a}(\mathrm{mK})$ & & 0.4 & 0.6 \\
\hline \hline
\end{tabular}

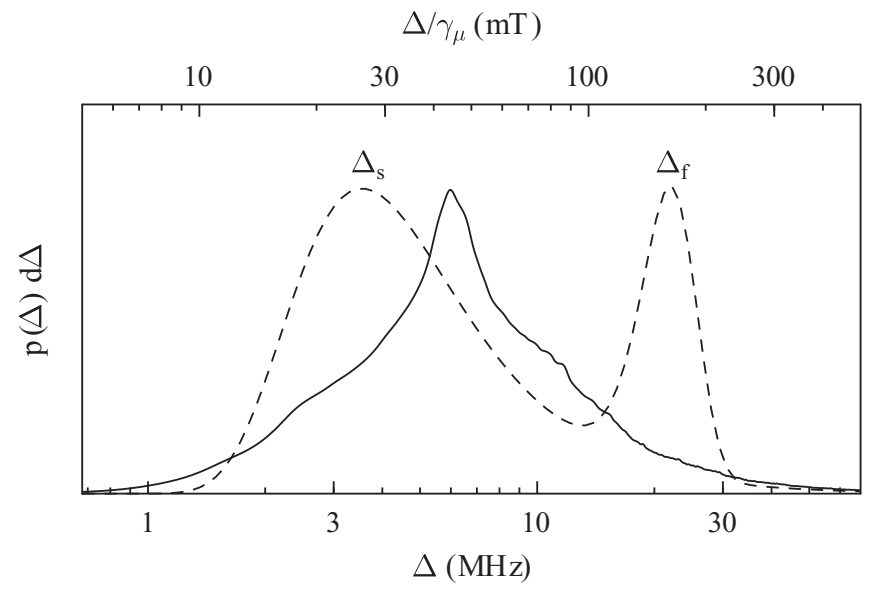

FIG. 6. The solid line shows the estimated probability distribution of the axis-projected local field $\Delta / \gamma_{\mu}$ for random muon stopping sites in the $(0,0,1 / 2)$ dipolar ordered state of $\mathrm{Fe}_{19}(\mathrm{~m})$. The dashed line illustrates the bimodal distribution inferred from the two observed relaxation components and their relaxation rates. This consists of a broad component $\left(\Delta_{\mathrm{s}}\right)$ with low coupling frequency and a narrow component $\left(\Delta_{\mathrm{f}}\right)$ with high coupling frequency.

\section{MUON RELAXATION COMPONENTS AND THE MUON SITE DISTRIBUTION}

From the knowledge of the most stable mode of ordering in $\mathrm{Fe}_{19}(\mathrm{~m})$, we can now look more closely at the form of the muon relaxation function and the origin of the subtle changes observed on going through the magnetic transition. A good starting point for this discussion is the distribution of internal fields for muons stopping randomly in the magnetic unit cell. This has been calculated using again the approximation of placing the full molecular spin at the center of the molecule. The obtained distribution is shown as the solid line in Fig. 6, where it is expressed in terms of $\Delta / \gamma_{\mu}$, which is the projection of the internal field onto a reference axis.

The distribution of $\Delta$ is broadly consistent with the average coupling of $39 \mathrm{mT}$ obtained previously [9] from the field dependence of the muon spin relaxation in $\mathrm{Fe}_{19}(\mathrm{e})$. The same study found that $v$, the residual fluctuation rate for the dynamic fields seen by the muon, was of order $100 \mathrm{MHz}$, in common with many similar SMM systems [23-25] where the residual electronic spin fluctuations at low temperatures are driven by hyperfine interactions. Since the $\Delta$ distribution lies well below $v$, the muon spin relaxation at all sites is in the fast fluctuation limit, where the muon spin relaxation rate at site $i$ is given by

$$
\lambda_{i}=2 \Delta_{i}^{2} / \nu \text {. }
$$

The observed relaxation function described by equation (1) indicates that the form of the actual $\Delta$ distribution in the system has two distinct regions (Fig. 6, dashed line). The slow root exponential relaxation is due to a broad low frequency region centered on $\Delta_{s}$, reflecting a group of stopping sites spread throughout the bulk of the molecule. In contrast, the fast exponential relaxation corresponds to a relatively narrow peak in the distribution centered on $\Delta_{\mathrm{f}}$, reflecting a group of stopping sites particularly close to the magnetic core of the molecule. The approximation of random stopping and a single point dipole works well for describing the low coupling region 

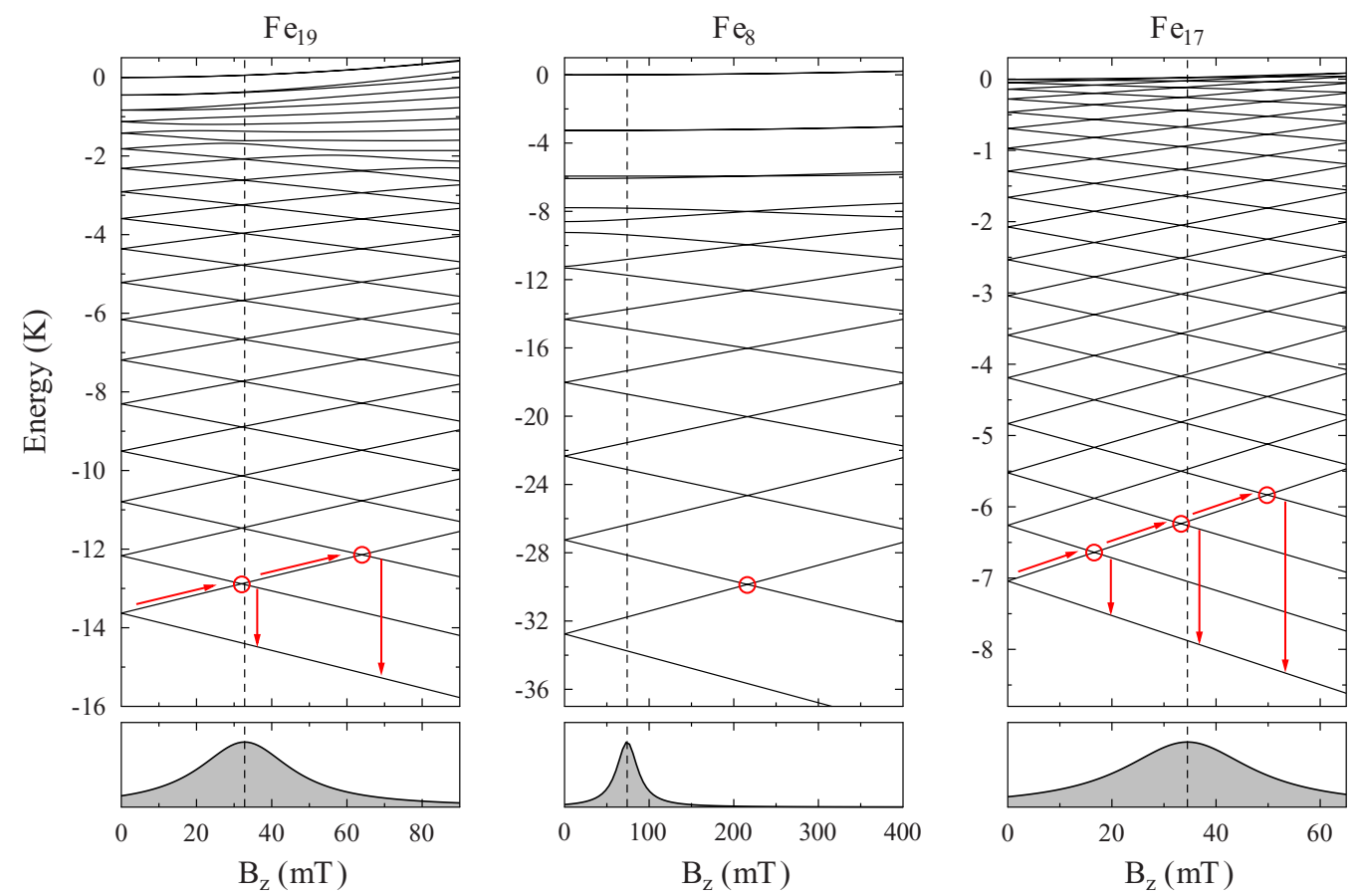

FIG. 7. (Color online) Comparison between the level structures and relaxation paths of $\mathrm{Fe}_{19}(\mathrm{~m}), \mathrm{Fe}_{8}$, and $\mathrm{Fe}_{17}$. The characteristic dipolar field bias produced by neighboring molecules when the system is close to order is indicated by the dashed line in each case; the lower panel includes a typical broadening seen in EPR [8], reflecting unresolved hyperfine structure from the large number of nuclear spins in the cell. Tunneling fields (marked with circles) are accessible without external field in the case of $\mathrm{Fe}_{19}$ and $\mathrm{Fe}_{17}$, thus providing efficient paths towards the ordered ground state (arrows). In contrast, internal fields for $\mathrm{Fe}_{8}$ are insufficient to reach a tunneling condition and the application of an external field is needed to reach an ordered state [5].

associated with $\Delta_{\mathrm{s}}$, but modeling the $\Delta_{\mathrm{f}}$ region is a much harder task, requiring knowledge of the exact muon stopping sites in the core region and taking into account the actual distribution of spin across the core.

The reason for the different behavior of the relaxation rates of the two relaxation components on going below $T_{\mathrm{c}}$ now becomes clear. For the fast component, $\Delta_{\mathrm{f}}$ is dominated by the adjacent spin core of the local molecule and is insensitive to the spins on neighboring molecules; the increase in $\lambda_{f}$ then simply reflects a drop of around $10 \%$ in $v$ on going through the transition. In contrast, the sites responsible for the slow component are closer to the edge of the molecule and therefore retain some sensitivity to the relative alignment of the spins of neighboring molecules. The onset of AF ordering then leads to a drop in the average field $\Delta_{\mathrm{s}} / \gamma_{\mu}$. A fall of around $10 \%$ in $\Delta_{\mathrm{s}}$ combined with the $10 \%$ drop in $v$ would then, following (4), lead to an overall fall of around $10 \%$ in $\lambda_{s}$, consistent with that observed experimentally.

\section{DIPOLAR BIAS AND THERMAL EQUILIBRIUM}

We now turn to the issue of thermal equilibrium versus spin freezing in these dipolar driven SMM systems in the absence of external magnetic fields. One of the key characteristics of the $\mathrm{Fe}_{19}$ system is that the separation between the two lowest levels of the $S_{z}$ multiplet is very similar to the dipolar energy, whereas this is not the case for the $\mathrm{Fe}_{8}$ system (see Table I). This has consequences for the level splitting of one molecule under the influence of the dipolar field provided by its neighbors, which is illustrated in Fig. 7. The dipolar field has the effect of biasing the $\mathrm{Fe}_{19}$ towards the first level-crossing resonance field and resonance can easily be achieved when residual broadening produced by factors such as hyperfine coupling to the many nuclear spins in the system is taken into account (Fig. 7, lower panels). The situation is even more favorable for $\mathrm{Fe}_{17}$, with the dipolar bias giving good access to three tunneling points. On the other hand, for $\mathrm{Fe}_{8}$, the dipolar bias draws the system away from the zero-field tunneling resonance, but is not large enough to reach any other resonance fields. The resonant tunneling rate is strongly dependent on having a field component transverse to the easy axis and this naturally occurs for the dipolar field in the tilted-disk triclinic structures of $\mathrm{Fe}_{19}$ (see $\phi$ in Table I). Since the changing of the magnetization direction of a molecule at low temperatures relies on these quantum tunneling processes, it can then be seen how a system such as $\mathrm{Fe}_{19}$ can easily find its equilibrium dipolar ordered ground state, whereas a system such as $\mathrm{Fe}_{8}$ needs careful preparation in field to achieve this [5].

\section{CONCLUSIONS}

In summary, $\mu \mathrm{SR}$ has observed a dipolar ordering transition in the $\mathrm{Fe}_{19}(\mathrm{~m}) \mathrm{SMM}$, a system that, having nearly 800 atoms in the ordered cell, is perhaps the magnetic system with the most complex structure yet studied using the $\mu \mathrm{SR}$ technique. The combination of a wide distribution of muon stopping sites and the rich internal dynamics of the SMM unit leads to a change in the muon spin relaxation function on ordering that is small but still measurable. Measurements of such subtle 
relaxation changes are more of an experimental challenge than the more familiar large amplitude precession signals of regular magnets. However, we note that the enhanced counting rates made possible by the latest muon instruments at pulsed sources such as ISIS and JPARC should be well suited to future studies of complex magnetic systems such as these.

The observed transition temperature for the dipolar ordering in $\mathrm{Fe}_{19}(\mathrm{~m})$ was found to be consistent with previous reports, with detailed modeling of the intermolecular dipolar coupling identifying the most stable mode of AF ordering. Monte Carlo simulations have confirmed that the observed transition temperature reflects the predominance of dipolar intermolecular interactions in this structure, although a minor contribution from superexchange interactions acting through the water of crystallisation can not be excluded.
A number of features of the $\mathrm{Fe}_{19} \mathrm{SMM}$ were found to be particularly favorable for rapidly achieving dipolar order. The large spin value combined with the moderate anisotropy leads to a dipolar bias field from surrounding molecules that is closely tuned to the first level crossing of the system and the triclinic crystal structure also gives the dipolar field a significant component transverse to the easy spin axis. These factors all enhance the quantum tunneling rate between spin states, enabling the system to avoid spin freezing at low temperatures and efficiently reach the equilibrium dipolar ordered state.

\section{ACKNOWLEDGMENT}

T.L. thanks the UK EPSRC and A.L. and P.A. thank the national FIRB project No. RBFR12RPD1 for support.
[1] D. Gatteschi, R. Sessoli, and J. Villain, Molecular Nanomagnets (Oxford University Press, New York, 2006).

[2] S. J. Blundell and F. L. Pratt, J. Phys.: Condens. Matter 16, R771 (2004).

[3] C. J. Wedge, G. A. Timco, E. T. Spielberg, R. E. George, F. Tuna, S. Rigby, E. J. L. McInnes, R. E. P. Winpenny, S. J. Blundell, and A. Ardavan, Phys. Rev. Lett. 108, 107204 (2012).

[4] A. Morello, F. L. Mettes, F. Luis, J. F. Fernández, J. Krzystek, G. Aromí, G. Christou, and L. J. de Jongh, Phys. Rev. Lett. 90, 017206 (2003).

[5] E. Burzuri, F. Luis, B. Barbara, R. Ballou, E. Ressouche, O. Montero, J. Campo, and S. Maegawa, Phys. Rev. Lett. 107, 097203 (2011).

[6] J. C. Goodwin, R. Sessoli, D. Gatteschi, W. Wernsdorfer, A. K. Powell, and S. L. Heath, J. Chem. Soc., Dalton Trans. 2000, 1835 (2000).

[7] E. Ruiz, A. Rodríguez-Forte, J. Cano, and S. Alvarez, J. Phys. Chem. Sol. 65, 799 (2004).

[8] L. Castelli, M. Fittipaldi, A. K. Powell, D. Gatteschi, and L. Sorace, Dalton Trans. 40, 8145 (2011).

[9] S. J. Blundell, F. L. Pratt, I. M. Marshall, C. A. Steer, W. Hayes, J.-F. Létard, S. L. Heath, A. Caneschi, and D. Gatteschi, Synth. Met. 133-134, 531 (2003).

[10] S. J. Blundell, F. L. Pratt, T. Lancaster, I. M. Marshall, C. A. Steer, S. L. Heath, J.-F. Létard, T. Sugano, D. Mihailovic, and A. Omerzu, Polyhedron 22, 1973 (2003).

[11] D. Procissi, A. Lascialfari, E. Micotti, M. Bertassi, P. Carretta, Y. Furukawa, and P. Kogerler, Phys. Rev. B 73, 184417 (2006).

[12] J. Lago, E. Micotti, M. Corti, A. Lascialfari, A. Bianchi, S. Carretta, P. Santini, D. Procissi, S. H. Baek, P. Kogerler, C. Baines, and A. Amato, Phys. Rev. B 76, 064432 (2007).

[13] M. Affronte, J. C. Lasjaunias, W. Wernsdorfer, R. Sessoli, D. Gatteschi, S. L. Heath, A. Fort, and A. Rettori, Phys. Rev. B 66, 064408 (2002).
[14] M. Affronte, R. Sessoli, D. Gatteschi, W. Wernsdorfer, J. C. Lasjaunias, S. L. Heath, A. K. Powell, A. Fort, and A. Rettori, J. Phys. Chem. Sol. 65, 745 (2004).

[15] M. Belesi, F. Borsa, and A. K. Powell, Phys. Rev. B 74, 184408 (2006).

[16] J. F. Fernandez and J. J. Alonso, Phys. Rev. B 62, 53 (2000); 65, 189901(E) (2002).

[17] F. L. Pratt, P. J. Baker, S. J. Blundell, T. Lancaster, M. A. Green, and M. Kurmoo, Phys. Rev. Lett. 99, 017202 (2007).

[18] T. Lancaster, S. J. Blundell, P. J. Baker, M. L. Brooks, W. Hayes, F. L. Pratt, R. Coldea, T. Sorgel, and M. Jansen, Phys. Rev. Lett. 100, 017206 (2008).

[19] K. Wieghardt, K. Pohl, I. Jibril, and G. Huttner, Angew. Chem. Int. Ed. 23, 77 (1984).

[20] F. L. Pratt, S. J. Blundell, T. Lancaster, C. Baines, and S. Takagi, Phys. Rev. Lett. 96, 247203 (2006).

[21] M. Evangelisti, A. Candini, A. Ghirri, M. Affronte, G. W. Powell, I. A. Gass, P. A. Wood, S. Parsons, E. K. Brechin, D. Collison, and S. L. Heath, Phys. Rev. Lett. 97, 167202 (2006).

[22] C. Vecchini, D. H. Ryan, L. M. D. Cranswick, M. Evangelisti, W. Kockelmann, P. G. Radaelli, A. Candini, M. Affronte, I. A. Gass, E. K. Brechin, and O. Moze, Phys. Rev. B 77, 224403 (2008).

[23] T. Lancaster, S. J. Blundell, F. L. Pratt, M. J. Brooks, J. L. Manson, E. K. Brechin, C. Cadiou, D. Low, E. J. L. McInnes, and R. E. P. Winpenny, J. Phys.: Condens. Matter 16, S4563 (2004).

[24] T. Lancaster, S. J. Blundell, F. L. Pratt, I. Franke, A. J. Steele, P. J. Baker, Z. Salman, C. Baines, I. Watanabe, S. Carretta, G. A. Timco, and R. E. P. Winpenny, Phys. Rev. B 81, 140409(R) (2010).

[25] A. Keren, O. Shafir, E. Shimshoni, V. Marvaud, A. Bachschmidt, and J. Long, Phys. Rev. Lett. 98, 257204 (2007).

[26] A.-L. Barra, P. Debrunner, D. Gatteschi, Ch. E. Schulz, and R. Sessoli, Europhys. Lett. 35, 133 (1996).

[27] M. Ueda, S. Maegawa, H. Miyasaka, and S. Kitagawa, J. Phys. Soc. Jpn. 70, 3084 (2001). 